\title{
REVISITING TUCKER'S ALGORITHM TO COLOR CIRCULAR ARC GRAPHS*
}

\author{
MARIO VALENCIA-PABON ${ }^{\dagger}$
}

\begin{abstract}
The circular arc coloring problem consists of finding a minimum coloring of a circular arc family $F$ such that no two intersecting arcs share a color. Let $l$ be the minimum number of circular arcs in $F$ that are needed to cover the circle. Tucker shows in [SIAM J. Appl. Math., 29 (1975), pp. 493-502], that if $l \geq 4$, then $\left\lfloor\frac{3}{2} L\right\rfloor$ colors suffice to color $F$, where $L$ denotes the load of $F$. We extend Tucker's result by showing that if $l \geq 5$, then $\left\lceil\left(\frac{l-1}{l-2}\right) L\right\rceil$ colors suffice to color $F$, and this upper bound is tight.
\end{abstract}

Key words. graph algorithms, chromatic number, circular arc graphs, induced cycles

AMS subject classifications. 05C $85,05 \mathrm{C} 15,05 \mathrm{C} 62,05 \mathrm{C} 38$

PII. S0097539700382157

1. Introduction. The circular arc coloring problem consists of finding a minimum coloring of a set of arcs of a circle such that no two intersecting arcs share a color. Applications include the problem of allocating bandwidth in all-optical WDM (wavelength division multiplexing) ring networks [5, 2], compiler design, and scheduling [9].

There are several results on the circular arc coloring problem $[1,9,4,6,7,3,5,2]$. Garey et al. [1] have shown that this problem is NP-hard, and Gavril shows in [3] that this problem remains NP-hard for Helly circular arc graphs, a subclass of circular arc graphs. Tucker [9] gave a simple 2-approximation algorithm and conjectured that $\frac{3}{2} \omega(F)$ colors are sufficient to color any family $F$ of arcs, where $\omega(F)$ represents the size of a maximum set of pairwise intersecting arcs in $F$. Karapetyan [4] shows Tucker's conjecture. Recently, Kumar [5] gave a randomized $(1+1 / e+o(1))$-approximation algorithm for instances of this problem needing at least $\omega(\ln n)$ colors, where $n$ is the number of arcs to be colored. For special cases of this problem, Orlin, Bonuccelli, and Bovet [6] found a $O\left(\mathrm{~m}^{2} \ln m\right)$ algorithm to color a family of $m$ proper circular arcs (i.e., no arc is contained in any other). The result of Orlin, Bonuccelli, and Bovet was improved by Shih and Hsu [7], who found a $O\left(\mathrm{~m}^{1.5}\right)$ algorithm to color a family of $m$ proper circular arcs. Recently, Gargano and Rescigno [2] show that a family of hereditary circular arcs can be optimally colored in polynomial time, and they show that there exists a $\frac{4}{3}$-approximation algorithm to color a larger class of families called quasi-hereditary families of arcs.

In this paper we give a tighter analysis of a greedy algorithm proposed by Tucker in [9] to color circular arc graphs.

The organization of the rest of the paper is as follows. In section 2 we give the preliminaries. Section 3 contains a tighter analysis of Tucker's greedy algorithm to color circular arc graphs, and section 4 contains concluding remarks.

\footnotetext{
${ }^{*}$ Received by the editors December 8, 2000; accepted for publication (in revised form) May 6, 2003 ; published electronically DATE. This work was supported by the Colombian OCyT (Observatorio Colombiano de Ciencia y Tecnología) and by COLCIENCIAS under grant 0006-2001.

http://www.siam.org/journals/sicomp/x-x/38215.html

†Departamento de Matemáticas, Universidad de los Andes, Cra. 1 No. 18A - 70, Bogotá, Colombia (mvalenci@uniandes.edu.co).
} 
2. Preliminaries. Let $F=\left\{A_{1}, A_{2}, \ldots, A_{m}\right\}$ be a family of circular arcs, where each $A_{i}$ represents an open real interval $\left(a_{i}, b_{i}\right)$ on a circle, with $a_{i}, b_{i} \in \mathbb{Z}^{+}$and $a_{i} \neq b_{i}$. Let $n$ denote the largest integer among all $a_{i}$ 's and $b_{i}$ 's. Then we can regard the circle as being divided into $n$ parts by $n$ equally spaced points numbered clockwise as $1,2, \ldots, n$, and each $A_{i}=\left(a_{i}, b_{i}\right)$ represents the circular arc from point $a_{i}$, which we call the beginning point of $A_{i}$, to point $b_{i}$, which we call the ending point of $A_{i}$, in the clockwise direction. We may assume that $n \leq 2 m$ (in the worst case, the beginning and ending points of any two arcs in $F$ are different).

Let $\chi(F)$ denote the minimum number of colors needed to color the arcs of $F$ such that no two intersecting arcs share a color, and let $\omega(F)$ denote the size of a maximum set of pairwise intersecting arcs in $F$. We call the load of $F$, which we denote by $L(F)$, the largest number or arcs containing a common point on the circle. Tucker shows in [9] that $\chi(F)$ verifies $L(F) \leq \chi(F) \leq 2 L(F)-1$. Note that $L(F)$ is not necessarily equal to $\omega(F)$. In [9] some families of arcs for which $\omega(F)=2 L(F)-1$ are given. Moreover, as $\chi(F) \geq \omega(F)$ holds for any family $F$ of arcs, Karapetyan's result [4] is the best deterministic approximation algorithm known for this problem. We denote by $\mathcal{G}(F)$ the intersection (undirected) graph corresponding to a circular arc family $F$, which is called a circular arc graph. Tucker [9] gives the following greedy algorithm to properly color a family $F$ of circular arcs.

TuCKeR's Algorithm.

1. Pick a point $p$ in the circle such that $S_{p}$, the set of arcs in $F$ containing $p$, has size $L(F)$. Let $A_{1}=\left(a_{1}, b_{1}\right)$ be the arc in $S_{p}$ that extends the least on the counterclockwise side of $p$, and let $c \max =1$. Assign color $c$ max to arc $A_{1}$.

2. For $i=1,2, \ldots,|F|-1$, we move clockwise round and round the circle indexing the arc $A_{i+1}=\left(a_{i+1}, b_{i+1}\right)$ in $F \backslash\left\{A_{1}, A_{2}, \ldots, A_{i}\right\}$ by the following rule: $A_{i+1}$ is the first unindexed arc to begin after the ending point of $A_{i}$. Assign color cmax to $A_{i+1}$ unless there exists an arc $A_{j}, j<i+1$, already colored with color cmax which intersects $A_{i+1}$, in which case we color $A_{i+1}$ with color $c \max +1$ and increment $c \max$.

In Figure 2.1 we show an example of the execution of Tucker's algorithm.

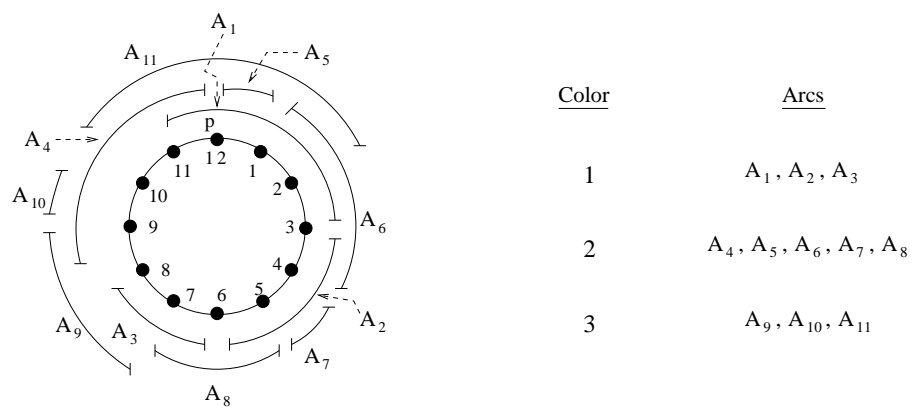

FIG. 2.1. Example of the execution of Tucker's algorithm.

Definition 2.1. Let $F$ be a family of circular arcs. We define the circular-cover of $F$ to be the smallest size of any subset of arcs of $F$ needed to cover the circle.

Using the previous algorithm, Tucker shows the following theorem, which we rephrase in terms of the circular-cover of a family of circular arcs.

TheOREm 2.2 (see Tucker [9]). Let $F$ be a family of circular arcs with load 
$L=L(F)$. If the circular-cover of $F$ is at least equal to four, then $\left\lfloor\frac{3}{2} L\right\rfloor$ colors suffice to color $F$.

Note that it must often be the case in practical situations that the circular-cover of a family of arcs is significantly greater than four. For instance, consider the task of assigning workers to jobs in a cyclic schedule. The time periods occupied by the jobs are the arcs, the colors assigned to the arcs are the workers, and the constraint that no worker can work on two jobs at once is realized by the constraint that no intersecting arcs can have the same color. The goal is to minimize the number of workers employed. Now, if the durations of the jobs are short compared with the period of the cyclic schedule, then the circular-cover of the corresponding family of arcs will be large. Therefore, in the following section, we analyze the performance of Tucker's greedy algorithm to color circular arc graphs in terms of the circular-cover of the associated family of arcs.

3. Analysis of Tucker's greedy algorithm. In this section we give a detailed analysis of Tucker's algorithm. Our main result is the following theorem, which extends Tucker's given in Theorem 2.2.

TheOREM 3.1. Let $F$ be a family of circular arcs with load $L=L(F)$. If the circular-cover of $F$ is equal to $l$, with $l \geq 5$, then $\left\lceil\left(\frac{l-1}{l-2}\right) L\right\rceil$ colors suffice to color $F$.

In order to prove Theorem 3.1, we need the following definitions. Let $F$ be a family of circular arcs with load $L(F)$, and assume that we use Tucker's greedy algorithm to color the arcs in $F$. Let $A_{1}=\left(a_{1}, b_{1}\right)$ be the first arc in $F$ considered by Tucker's algorithm. Let us denote the beginning point of $\operatorname{arc} A_{1}$ as $t$. We say that Tucker's algorithm has completed $k$ rounds if the algorithm has traversed the point $t k+1$ times, where arc $A_{1}$ counts. We let $T_{k}$ be the set of arcs colored during the $k$ th round, and we let $F_{k}=\bigcup_{i=1}^{k} T_{i}$ be the subfamily of arcs colored until the $k$ th round.

Proof of Theorem 3.1. Let $m$ be the largest integer used in the description of the $\operatorname{arcs}$ in $F$. W.l.o.g. assume that each two consecutive points $p$ and $p+1$ on the circle, $1 \leq p \leq m$, are traversed by exactly $L$ arcs from $F$. Otherwise, if some two consecutive points $p$ and $p+1$ are traversed by $r<L$ arcs, then we add $L-r$ arcs of the form $(p, p+1)$ (or $(m, 1)$ if $p=m$ ) to $F$ without changing its colorability. We assume that we use Tucker's algorithm to color the $\operatorname{arcs}$ in $F$, and we proceed by induction on $L$. If $L=1$, the result is trivial. Assume that the result holds when $L(F)<L$ for any $F$ and some $L \geq 2$, and we shall prove that the result holds for any $F$ with $L(F)=L$. By hypothesis, the circular-cover $l$ of $F$ verifies $l \geq 5$. Let $A_{1}$ be the first arc in $F$ colored by Tucker's algorithm, and let $t$ be the beginning point of arc $A_{1}$. For $i=1,2, \ldots$, let $A_{i}$ denote the first arc in $F$ colored $i$. Thus, considering the way Tucker's algorithm colors the arcs in $F$, we can deduce the following properties. We prove only Property 3 , because Properties 1 and 2 can be easily deduced.

Property 1. For each $i, i>1, A_{i}$ intersects $A_{i-1}$.

Property 2. For each $i, i \geq 1, L\left(F \backslash F_{i}\right) \leq L(F)-i$, where $L\left(F \backslash F_{i}\right)$ is the load induced by the subfamily of arcs $F \backslash F_{i}$.

Property 3. For each $i, 1 \leq i \leq l-2$, Tucker's algorithm uses at most $i+1$ colors to color the arcs in $F_{i}$, where $l$ is the circular-cover of the family $F$.

Proof of Property 3. We proceed by induction on $i$. If $i=1$, the result is trivial. Assume that the result holds for $j<i$, and we will prove that the result holds for $j=i$. Let $i>1$. Let $A_{i}$ be the first arc in $T_{i-1}$ colored with color $i$, and let $A_{i}^{f}$ be the last arc in $T_{i-1}$ colored with color $i$ that traverses the point $t$ for the $i$ th time (note that $A_{i}$ and $A_{i}^{f}$ can be the same arc (see Figure 3.1(b)). By induction hypothesis, these arcs exist; otherwise, the number of colors used by the algorithm to color the 
arcs in $F_{i-1}$ is at most $i-1$, and the result trivially follows. Now, let $A_{i+1}$ be the first arc in $T_{i}$ colored with color $i+1$. By Property 1 , we have that $A_{i+1}$ intersects $A_{i}$. Let $A_{i+1}^{f}$ be the last arc in $T_{i}$ that traverses the point $t$ for the $(i+1)$ th time. Suppose that $A_{i+1}^{f}$ cannot be colored with color $i+1$. Thus, by Property 1 , we have that $A_{i+1}$ intersects $A_{i+1}^{f}$, and so the $i+1 \operatorname{arcs} A_{2}, A_{3}, \ldots, A_{i}, A_{i+1}, A_{i+1}^{f}$ cover the circle (see Figure 3.1(c)). However, $i \leq l-2$, which implies that the circular-cover of $F$ is at most $l-1$, which is a contradiction. This ends the proof of this property.

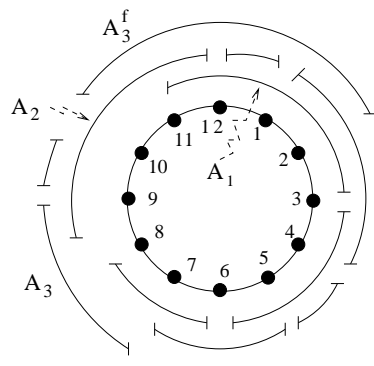

(a)

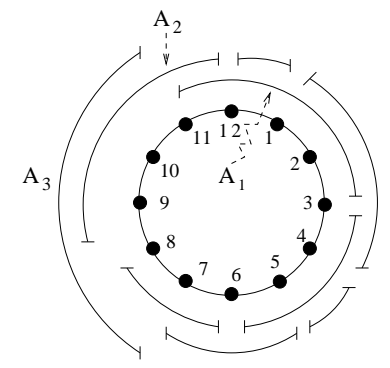

(b)

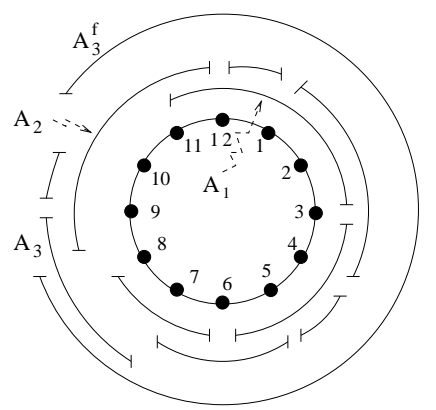

(c)

FIG. 3.1. (a), (b) Examples of possible configurations in the proof of Property 3 for $i=2$ and $l=7$. (c) Example of forbidden configuration in the proof of Property 3 for $i=2$ and $l=7$.

Now, the proof of Theorem 3.1 can be easily deduced from Properties 2 and 3 as follows. By Property 2, we have that $L\left(F \backslash F_{l-2}\right) \leq L-(l-2)$ and, by Property 3 , we have that Tucker's algorithm uses at most $l-1$ colors to color the arcs in $F_{l-2}$. Therefore, by induction hypothesis, the theorem holds.

The following corollary can be deduced directly from Theorem 3.1.

Corollary 3.2. Let $F$ be a family of circular arcs with load $L=L(F)$ on a circle on $n$ points. For all $k \geq 3$, if each arc in $F$ spans at most $n / k$ points of the circle, then the number of colors used by Tucker's algorithm to color $F$ is at most $\left\lceil\left(\frac{k}{k-1}\right) L\right\rceil$.

It is easy to verify that in the complementary case of Corollary 3.2 , i.e., when each circular arc in a family $F$ spans more than half of the points in a circle, the intersection graph of the arcs in $F$ is a complete graph on $|F|$ vertices.

Proposition 3.3. Let $F$ be a family of circular arcs on a circle. If each arc in $F$ spans more than half of the points in the circle, then $F$ can be optimally colored in linear time.

The following proposition gives us an idea of the tightness of the upper bound obtained in Theorem 3.1.

Proposition 3.4. For any odd integer $l, l \geq 3$, and for any even integer $L$, $L \geq 2$, there exists a family $F$ of circular arcs with load $L$ and circular-cover $l$ such that $\chi(F)=\left\lceil\left(\frac{l}{l-1}\right) L\right\rceil$.

Proof. In order to prove Proposition 3.4, we use a result of Stahl [8] concerning the $r$-tuple colorings of graphs. An $r$-tuple coloring of a graph $G$ is an assignment of $r$ distinct colors to each vertex of $G$ in such a manner that no two adjacent vertices share a color. In the special case of a cycle $C_{l}$ with odd length $l, l \geq 3$, Stahl shows in [8] that the minimum number of colors for a $r$-tuple coloring of $C_{l}, r \geq 1$, is equal to 
$2 r+1+\left\lfloor\frac{2(r-1)}{l-1}\right\rfloor$. Thus, $F$ is constructed as follows. Let $r=L / 2$, and assume that the vertices of $C_{l}$ are labeled clockwise by $0,1, \ldots, l-1$. For all $i=0,1, \ldots, l-1, F$ contains $r$ copies of the arc $(i, i+2 \bmod l)$. By construction, it is easy to verify that if $L=2$ (and thus $r=1$ ), then the intersection graph of the $\operatorname{arcs}$ in $F$ is isomorphic to $C_{l}$ and therefore the circular-cover of $F$ is equal to $l$. Hence, for any even integer $L=2 r$, with $r \geq 1$, there exists a family $F$ of circular arcs with load $L$ and circular-cover $l$ such that $\chi(F)=2 r+1+\left\lfloor\frac{2 r-2}{l-1}\right\rfloor=L+1+\left\lfloor\frac{L-2}{l-1}\right\rfloor=\left\lceil\left(\frac{l}{l-1}\right) L\right\rceil$.

Note that we cannot hope that, for any family $F$ with load $L$ and circular-cover $l \geq 5,\left\lfloor\left(\frac{l-1}{l-2}\right) L\right\rfloor$ colors suffice to color the arcs in $F$. In fact, by Proposition 3.4, we have that any cycle graph $C_{l}$ with odd length $l, l \geq 5$, can be represented by a family of $l$ arcs with load 2 and circular-cover equal to $l$. Thus, $\left\lfloor\left(\frac{l-1}{l-2}\right) 2\right\rfloor=2$, but the chromatic number of $C_{l}$ is 3 . The following proposition shows that there are instances of the circular arc coloring problem where the upper bound in Theorem 3.1 is reached.

Proposition 3.5. The upper bound in Theorem 3.1 is tight.

Proof. Let $C_{l}$ be a cycle graph on $l$ vertices, with $l=2 k+1$ for any positive integer $k \geq 2$. By Proposition 3.4, we have that for all $r, 1 \leq r \leq k-1$, an $r$ tuple coloring of $C_{l}$ can be represented by a family $F$ of arcs with load $L=2 r$ and circular-cover $l$. Moreover, the minimum number of colors needed to color $F$ is exactly $2 r+1$. By Theorem 3.1, we have that the $\operatorname{arcs}$ in $F$ can be colored using at most $\left\lceil\left(\frac{l-1}{l-2}\right) L\right\rceil=\left\lceil\frac{4 k r}{2 k-1}\right\rceil=\left\lceil 2 r+\frac{2 r}{2 k-1}\right\rceil=2 r+1$ colors. This ends the proof of this proposition.

Let $F$ be a family of arcs with load $L(F)$. By Property 2 in Theorem 3.1, we have that for each $i, i \geq 1$, the load of the subfamily $F \backslash F_{i}$ is at most equal to $L(F)-i$. Moreover, for each round $i$, Tucker's algorithm uses at most two new colors to color the $\operatorname{arcs}$ in $T_{i}$. Therefore, in the general case (i.e., if the circular-cover $l$ of $F$ verifies $l<4)$, the number of colors used by Tucker's algorithm to color the arcs in $F$ is at most equal to $2 L(F)$, and thus it gives a 2-approximation algorithm for the circular arc coloring problem, as noted earlier by Tucker in [9].

4. Conclusion. We have shown a tighter analysis of Tucker's greedy algorithm to color a family of circular arcs. We have proved that Tucker's algorithm is quasioptimal for families of arcs having a large circular-cover. Our results extend a previous one given by Tucker. An interesting open problem is the complexity of the circular arc coloring problem for families of arcs having bounded spans and unbounded load.

Acknowledgment. The author gratefully acknowledges the helpful comments and suggestions of two anonymous referees.

\section{REFERENCES}

[1] M. R. Garey, D. S. Johnson, G. L. Miller, and C. H. Papadimitriou, The complexity of coloring circular arcs and chords, SIAM J. Alg. Disc. Meth., 1 (1980), pp. 216-227.

[2] L. Gargano and A. Rescigno, Coloring circular arcs with applications to WDM routing, in Proceedings of the Workshop on Approximation and Randomization Algorithms in Communication Networks, Geneva, Switzerland, 2000.

[3] F. GavriL, Intersection graphs of Helly families of subtrees, Discrete Appl. Math., 66 (1996), pp. $45-56$.

[4] I. A. Karapetyan, Coloring of arc graphs, Akad. Nauk Armyan. SSR Dokl., 70 (1980), pp. 306311 (in Russian).

[5] V. KumAR, An approximation algorithm for circular arc coloring, Algorithmica, 30 (2001), pp. 406-417.

[6] J. B. Orlin, M. A. Bonuccelli, and D. P. Bovet, An $O\left(n^{2}\right)$ algorithm for coloring proper circular arc graphs, SIAM J. Alg. Disc. Meth., 2 (1981), pp. 88-93. 
[7] W.-K. Shin And W.-L. Hsu, An $O\left(n^{1.5}\right)$ algorithm to color proper circular arcs, Discrete Appl. Math., 25 (1989), pp. 321-323.

[8] S. StAhL, $n$-tuple colorings and associated graphs, J. Combinatorial Theory Ser. B, 20 (1976), pp. 185-203.

[9] A. Tucker, Coloring a family of circular arcs, SIAM J. Appl. Math., 29 (1975), pp. 493-502. 